Published in final edited form as:

Hepatology. 2014 September ; 60(3): 896-907. doi:10.1002/hep.27050.

\title{
Human bile contains microRNA-laden extracellular vesicles that can be used for cholangiocarcinoma diagnosis
}

\author{
Ling $\mathrm{Li}^{1,2}$, David Masica ${ }^{3}$, Masaharu Ishida ${ }^{1}$, Ciprian Tomuleasa ${ }^{1,4}$, Sho Umegaki ${ }^{5}$, Anthony \\ N. Kalloo ${ }^{1}$, Christos Georgiades ${ }^{6,7}$, Vikesh K. Singh ${ }^{1}$, Mouen Khashab ${ }^{1}$, Stuart Amateau ${ }^{8}$, \\ Zhiping Li ${ }^{1}$, Patrick Okolo ${ }^{1}$, Anne-Marie Lennon ${ }^{1}$, Payal Saxena ${ }^{1}$, Jean-Francois \\ Geschwind $^{6}$, Todd Schlachter ${ }^{6}$, Kelvin Hong ${ }^{6}$, Timothy M. Pawlik ${ }^{9}$, Marcia Canto ${ }^{1}$, Joanna \\ Law $^{1}$, Reem Sharaiha ${ }^{10}$, Clifford R. Weiss ${ }^{6}$, Paul Thuluvath ${ }^{11}$, Michael Goggins ${ }^{1}$, Eun Ji \\ Shin ${ }^{1}$, Haoran Peng ${ }^{1}$, Vivek Kumbhari ${ }^{1}$, Susan Hutfless ${ }^{1}$, Liya Zhou ${ }^{2}$, Esteban Mezey ${ }^{1}$, \\ Stephen J. Meltzer ${ }^{1}$, Rachel Karchin ${ }^{3}$, and Florin M. Selaru1,12, ${ }^{\text {}}$ \\ ${ }^{1}$ Division of Gastroenterology and Hepatology, Department of Medicine, Johns Hopkins Hospital, \\ Baltimore, Maryland, USA \\ 2Division of Gastroenterology, Third hospital of Peking University Health Science Center, Beijing, \\ China \\ ${ }^{3}$ Department of Biomedical Engineering and Institute for Computational Medicine Johns Hopkins \\ University, Baltimore, Maryland, USA \\ ${ }^{4}$ Center for Genomics and Translational Medicine, Iuliu Hatieganu University of Medicine and \\ Pharmacy, and Department of Hematology, Ion Chiricuta Comprehensive Cancer Center, Cluj \\ Napoca, Romania \\ ${ }^{5}$ Tohoku University School of Medicine, Sendai, Miyagi, Japan \\ ${ }^{6}$ Division of Gastroenterology and Hepatology, Department of Radiology, Johns Hopkins Hospital, \\ Baltimore, Maryland, USA \\ ${ }^{7}$ Vascular \& Interventional Radiology, American Medical Center, Nicosia, Cyprus \\ ${ }^{8}$ Division of Gastroenterology and Hepatology, University of Colorado, Denver, Colorado, USA \\ ${ }^{9}$ Division of Gastroenterology and Hepatology, Department of Surgery, Johns Hopkins Hospital, \\ Baltimore, Maryland, USA \\ ${ }^{10}$ Division of Gastroenterology and Hepatology, Weill Cornell Medical College, New York, USA \\ ${ }^{11}$ The Institute for Digestive Health \& Liver Disease at Mercy, Baltimore, USA \\ ${ }^{12}$ Sidney Kimmel Cancer Center, Johns Hopkins Hospital, Baltimore, Maryland, USA
}

*Correspondence: Florin M. Selaru, MD, Johns Hopkins University, 720 Rutland Ave, Suite 950, Tel: (410) 614-3369, Fax: (410) 614-9612, selaru@jhu.edu.

Disclosures: No authors have any potential conflicts in reference to this manuscript

Author contribution: Study concept and design (FMS, LL), acquisition of data (LL, DM, CT, SU,MI, ANK, ZL, VKS, MK, PO, AML,PS, TMP,CG, JFG, SA, TS, KH, MC, JL, RS, CRW, EJS, PT, MG,VK, SH, LZ, EM, SJM, RK, FMS,), analysis and interpretation of data (LL, DM, FSM), drafting of the manuscript (LL, DM), critical revision of the manuscript (VKS, TMP, SA, SH, EM, SJM, RK, FMS), statistical analysis (DM, SH, FSM, RK, LL). 


\section{Abstract}

Cholangiocarcinoma (CCA) presents significant diagnostic challenges, resulting in late patient diagnosis and poor survival rates. Primary Sclerosing Cholangitis (PSC) patients pose a particularly difficult clinical dilemma, since they harbor chronic biliary strictures that are difficult to distinguish from CCA. MicroRNAs (miRs) have recently emerged as a valuable class of diagnostic markers; however, thus far, neither extracellular vesicles (EVs) nor miRs within EVs have been investigated in human bile. We aimed to comprehensively characterize human biliary EVs, including their miR content.

Conclusion-We have established the presence of extracellular vesicles in human bile. In addition, we have demonstrated that human biliary EVs contain abundant miR species, which are stable and therefore amenable to the development of disease marker panels. Furthermore, we have characterized the protein content, size, numbers and size distribution of human biliary EVs. Utilizing Multivariate Organization of Combinatorial Alterations (MOCA), we defined a novel biliary vesicle miR-based panel for CCA diagnosis which demonstrated a sensitivity of $67 \%$ and specificity of $96 \%$. Importantly, our control group contained 13 PSC patients, 16 patients with biliary obstruction of varying etiologies (including benign biliary stricture, papillary stenosis, choledocholithiasis, extrinsic compression from pancreatic cysts, and cholangitis), and 3 patients with bile leak syndromes. Clinically, these types of patients present with a biliary obstructive clinical picture that could be confused with CCA. These findings establish the importance of using extracellular vesicles, rather than whole bile, for developing miR-based disease markers in bile. Finally, we report the development of a novel bile-based CCA diagnostic panel that is stable, reproducible, and has potential clinical utility.

\section{Keywords}

extracellular vesicles; cholangiocarcinoma; microRNA

\section{Introduction}

Cholangiocarcinoma (CCA) is a cancer that arises in the biliary tree (1). Anatomically, CCA is divided into intrahepatic (iCCA), perihilar (pCCA) and distal (dCCA) tumors (2). Surgery is the only curative option (3). Unfortunately, due to the nonspecific nature of symptoms, as well as to failure of currently available tests, patients are usually diagnosed late in disease progression, when they are no longer surgical candidates (3). All three types of CCA present diagnostic dilemmas. For example, the diagnosis of iCCA is, in part, based on lack of liver cirrhosis and absence of any other known primary solid tumors (2). However, iCCA can also develop in cirrhotic livers, and a small size iCCA arising in a cirrhotic liver may mimic HCC in terms of its rapid uptake of contrast material (4). Diagnosing pCCA is equally difficult, despite of a variety of available diagnostic tools, including magnetic resonance imaging (MRI), computed tomography (CT), endoscopic retrograde cholangiopancreatography (ERCP), cholangiosccopy and endoscopic ultrasound (EUS). pCCA tends to display a strong desmoplastic reaction, which poses a significant diagnostic challenge, since obtaining cells from these lesions for cytologic examination is exceedingly difficult (3). Therefore, the sensitivity of cytology performed on brush biopsy specimens is, at best, only $20 \%$ (2). Similar to pCCA, cancers located in the distal bile duct (dCCA) 
display low celullarity and a strong desmoplastic reaction, rendering cytologic diagnosis very difficult.

Multiple recent studies have sought to develop more precise markers of CCA. One such approach aimed at diagnosing CCA based on serum proteomics (5). Additional studies have focused on RNA expression profiles in biliary brushings (6) or on microRNA profiles in whole human bile (7). However, there are several limitations in interpreting the results from these studies. First, these studies tended to include small numbers of patients. In addition, standardization of specimen collection, specimen manipulation and marker derivation have received inadequate attention. For example, in previous bile-based studies, there has been scant information regarding standardization of bile processing to ensure reproducible and reliable results. The issue of unreliable and/or conflicting results is paramount in cancer marker development (8). Therefore, it is not surprising that studies published to date present contradictory information regarding specific miR-based markers of cancer (9). In addition to which methodologies are best for body fluid collection, storage and processing, there are several unanswered questions, including the appropriate reference gene(s) for normalization. Serum/plasma studies thus far have employed a variety of reference genes, including miR-16, miR-142-3p, let-7a, and small RNA U6. While it is difficult to predict which of these RNAs serves as the best the normalizer, it is apparent that some are worse than others. U6, in particular, which is approximately 4 times longer than any miR, should be avoided in miR-based marker panels in biologic fluids because it is less stable than miR species in body fluids and displays a different dynamic of degradation $(10,11)$. Unfortunately, the only previous bile-based miR panel for CCA diagnosis employed U6 as a normalizer (7). Lastly, given the complex makeup of biologic fluids, it is naïve to hypothesize that a single RNA exhibits constant expression across various physiologic and pathologic states. Recent evidence suggests that in order to circumvent the need for an internal control, synthetic miR sequences can be spiked into biologic fluids prior to RNA extraction (12).

We hypothesized that because CCAs are in direct contact with bile, an accurate tumorderived miR profile is more likely to exist in bile than in serum. In the current study, we present analyses of human bile geared toward developing a reliable, reproducible miR-based CCA diagnostic panel. We investigated the source of miRs in human bile, the stability of miR profiles in human bile, the best bile processing procedures, and the most stable miR panel for diagnosing CCA from human bile.

\section{Materials and Methods}

\section{Bile samples}

Bile samples from CCA and control (CTRL) patients were obtained from the Johns Hopkins Hospital, under an IRB approved protocol. Bile samples were obtained at ERCP or at the time of percutaneous manipulation of biliary tubes by interventional radiology (IR). Aspiration of bile was performed after cannulation of the biliary tree prior to injection of contrast. The CCA diagnosis was established based on pathologic and radiologic evidence.Table 1A contains demographic and clinical patient information.Table 1B contains the TNM stage for CCA patients who had carbohydrate antigen 19-9 (CA19-9) measured. Numerous patients with benign biliary obstruction were included in the control group. These 
patients were added because, from a clinical perspective, they present with a clinical picture indistinguishable from CCA. Thus, our marker panel was envisioned as particularly useful in this setting. Supplementary Table 1 provides detailed information regarding control patients. For example, there were 13 patients with PSC. These patients were followed for 5 years after bile specimens were collected, to ensure that they were not already harboring early undiagnosed CCA. In addition, we included 15 patients with benign biliary tree obstruction and 3 patients with benign bile leaks.

\section{Bile extracellular vesicle isolation}

Initially, we experimented with EVs isolation from 1 milliliter $(\mathrm{mL})$ of fresh bile. Once the experimental procedures were carefully delineated, we started extracting EVs from 400 microliters $(\mu \mathrm{L})$ of bile. The bile samples were centrifuged at $300 \mathrm{Gs}$ for $10 \mathrm{~min}$ at 4 degrees Celsius $\left({ }^{\circ} \mathrm{C}\right)$ to pellet cells and debris. The supernatant was then centrifuged at 16,500 Gs for $20 \mathrm{~min}$ at $4{ }^{\circ} \mathrm{C}$ to further remove cellular debris and then filtered through a 200 nanometer (nm) filter. Next, the supernatant was centrifuged at $120,000 \mathrm{Gs}$ for 70 minutes at $4{ }^{\circ} \mathrm{C}$ to pellet EVs (13). The EVs were utilized for immediate RNA extraction or resuspended in 50$150 \mu \mathrm{L}$ PBS and stored in $-80^{\circ} \mathrm{C}$ for future use.

\section{Statistical analyses}

We used three computational packages to assess the predictive value of selected miR species for CCA diagnosis: Random Forests (RF), Support Vector Machines (SVMs), and our recently developed MOCA algorithm (see details below) $(14,15)$. For details, please see Supplementary Methods.

\section{Supplementary Materials}

For detailed Materials and Methods, please see supplementary materials, to include Supplementary Figures 1 and 2 and Supplementary Table 2.

\section{Results}

\section{Delineation of the source of miRs in human bile}

The sole published report utilizing human bile for miR-based CCA diagnostic panels employed whole bile (7). Based on RNA gel electrophoresis, as well as qRT-PCR values for a well expressed miR species (miR-21), we determined that free floating cells in bile contribute to the RNA extracted from bile (Supplementary Figure 3A). Unfortunately, the quantity and quality of the RNA contributed by free floating cells likely depends on the number of free floating cells in a specific bile specimen and also on the degree of cell viability, and therefore is unpredictable. In addition, we demonstrate that the RNA contributed by free floating cells is rapidly degraded at RT, as well as due to a single freezethaw cycle (Supplementary Figure 3A and 3B). These data strongly argue against using whole bile for developing a miR-based disease marker panel. 


\section{Isolation and characterization of human bile extracellular vesicles}

Extracellular vesicle preparations from human bile were imaged by using TEM. We noted the presence of 30-110 nanometer (nM) vesicles, consistent with previously reported features of EVs (Figure 1A) (16). To further confirm that these spherical structures are EVs, we assayed for presence of Tumor Susceptibility Gene 101 (TSG101) and CD63, molecules frequently used as extracellular vesicle markers (17). Western blotting confirmed that the biliary derived extracellular vesicle preparations are rich in TSG101 and CD63, as shown in Figure 1B. To further define human biliary EVs, we employed multi-parameter NTA. First we noted the presence of round vesicles, displaying typical Brownian motion (Figure 1C and Supplementary Movie 1). Next, we found that the majority of EVs in human bile were between $30-110 \mathrm{nM}$ and that the mode of extracellular vesicle sizes was $84 \mathrm{nM}$ (Figure 1D), suggesting that EVs isolated are most likely exosomes. Based on NTA analysis, we also determined that the bile from CCA patients contained approximately $3 X 10^{\wedge} 11 \mathrm{EVs} / \mathrm{mL}$ bile, and bile from control patients contained approximately $2.5 \times 10^{\wedge} 10 \mathrm{EVs} / \mathrm{mL}$ bile. To further substantiate the presence of EVs in preparations from human bile, we stained EVs with PKH67, as previously described (18). Next, we added stained EVs or control extracts to cells in culture. Cells took up stained EVs from human bile but not from the controls, as shown in Supplementary Figure 4 and Supplementary Movie 2.

\section{Presence and isolation of miR species from biliary extracellular vesicles}

EVs isolated from serum were demonstrated to contain miR species, but no studies to date investigated human bile EVs (19). We performed qRT-PCR miR arrays on extracellular vesicle RNA isolated from 1 CCA bile specimen (Figure 2A). According to the manufacturer's recommendation, we utilized a $\mathrm{Ct}$ value threshold of 40 cycles to establish which miR species existed in quantities high enough to be detected. Utilizing a Ct value threshold of 40 cycles, we were able to detect $137 \mathrm{miR}$ species. From these $137 \mathrm{miR}$ species with amplification, a number of $74 \mathrm{miR}$ species amplified at a Ct value of 32 or less, which is considered by the manufactured as reliable amplification.

\section{Identification of a normalizer for miR species extracted from biliary extracellular vesicles}

Starting with the same initial volume of bile, utilization of such a synthetic miR will normalize for any variability in RNA extraction. To gain insight into the potential presence and/or magnitude of such biases, we spiked Cel-miR-39 into EVs extracted from 60 human bile specimens. Next, we extracted RNA from these specimens and performed qRT-PCR for Cel-miR-39. As Figure 2B demonstrates, the measured quantity of Cel-miR-39 differed dramatically across the 60 samples. The comparison between the highest and the lowest measured quantity of Cel-miR-39 revealed a dynamic range of 65 -fold. Based on these data, we concluded that normalizing for RNA extraction efficiency by spiking Cel-miR-39 into all specimens is mandatory.

\section{Stability of miR species in biliary extracellular vesicles}

We aimed at determining the stability of miR species extracted from biliary EVs. To determined the effects of storing bile specimens at RT or freeze-thaw cycles, we chose 2 miR species, miR-21 and miR-638, that in our previous experiments were found to be 
expressed in biliary EVs. Preliminary experiments on CCA tissues (not presented here) demonstrated good expression of miRs -21 and -638 . We first verified that high tissue expression translated into measurable levels in bile. Next, we utilized primers for these 2 miR species to perform pilot experiments in bile in order to document reproducibility, as well as to assess the best bile and extracellular vesicle handling techniques. As Figure 3 demonstrates, storing whole bile at RT for up to $48 \mathrm{~h}$ and up to 3 freeze-thaw cycles has a negligible effect onto expression of human bile extracellular vesicle content of miR species.

\section{Identification of differentially expressed miR species between biliary EVs of CCA and control patients}

qRT-PCR miR arrays were performed on a total of 6 specimens (RNA extracted from EVs isolated from $3 \mathrm{CCA}$ and 3 control bile specimens). There were $54 \mathrm{miR}$ species which had amplification at $\mathrm{Ct}$ of 32 or less in at least $2 \mathrm{CCA}$ specimens. The average normalized value for each miR species was calculated for CCA and control specimens, respectively. The fold ratio of miR expression in CCA vs. control specimens was utilized to order these miR species. We then selected the top $11 \mathrm{miR}$ species (Table 2 and Figure 4) for further analyses.

\section{Comparison of mathematical models to analyze extracellular vesicle miR profiles}

To assess the predictive value of selected miR species for CCA diagnosis we used three distinct mathematical approaches: RFs, SVMs, and MOCA algorithm (see Materials and Methods $)(14,15)$. Table 3A shows the sensitivity and specificity for diagnosing CCA from selected miR species using each of the three mathematical approaches. For each approach, the miR species facilitate reasonably accurate CCA diagnosis. The sum of sensitivity and specificity is approximately equal for MOCA and RFs, with RFs achieving greater sensitivity ( $76 \%$ vs. $67 \%$ ) and MOCA achieving greater specificity ( $96 \%$ vs. $88 \%)$. SVMs achieved a slightly greater specificity than RFs (90\% vs. $88 \%$ ), but in general MOCA and RFs outperformed SVMs. MOCA is unique among the three methods in that it selects biomarkers that can be used for subsequent clinical diagnosis, whereas the other methods generate black-box models that are highly dependent on the training data. Furthermore, biomarkers selected by MOCA have potential clinical utility, because the corresponding thresholds for CCA diagnosis are well above the sample means (Table 3B); with SVMs and RFs there is no guarantee that samples were partitioned using expression values within the resolution of the experiment. Because MOCA predictions were consistent, provide a clear biological relationship between predictors and classification, and distinguish cancers from controls with clinically relevant resolution, we performed all further analyses using MOCA results.

\section{Utilization of MOCA for human CCA diagnosis based on bile extracellular vesicle miR expression}

Table 3C shows representative, highly predictive biomarkers of CCA that comprised five, four, three, or two species, and the corresponding statistical sensitivity and specificity. All biomarkers comprising two or more miR species are the result of combining those constituent miRs using the union Boolean set operation (see Materials and Methods). Several of the markers that combine six miRs have predictive values equal to that of the first

Hepatology. Author manuscript; available in PMC 2015 September 01. 
marker in Table 3C (marker 1); however, marker 1 is subset of any higher-order marker that has an equivalent predictive value, and therefore use of these high-order markers is superfluous and not considered here. Markers combining more than six miRs have an overall decreasing predictive value owing to a substantial decrease in specificity. Conversely, lower-order markers (those combining three, two, or a single miR species) had decreasing predictive value owing to a decrease in sensitivity (Table 3C). For example, the marker comprising miR-191, miR-486-3p, and miR-1274b has a sensitivity and specificity of 59\% and $96 \%$, respectively. The marker combining miR-191 and miR-486-3p is has a sensitivity of $57 \%$ and a specificity of $96 \%$. No single miR marker consistently passed the 0.05 FDR during 10-fold cross validation, and was not considered further.

The distribution of CCA classification for the four representative multi-miR markers from Table 3C, and expression of each miR for the corresponding individual markers is shown in Figure $5 \mathrm{~A}$ and $5 \mathrm{~B}$. This representation is useful to determine when a marker uniquely diagnoses CCA, for which samples there is a consensus of diagnoses, and which markers complement to create highly predictive multi-miR biomarkers. For instance, miR-486-3p is unique among the five miRs in that it is the only miR that accurately diagnoses samples CCA23 36, 39, and 43. Similarly, miR-191 is the only marker that accurately -diagnoses CCA1 and 46; taken together, this complementarity explains why miRs-191 and miR-486-3p combine to make a highly predictive two-miR marker. Similarly, miR-1274b is unique in accurately classifying $C C A 6$ and 37 , which explains why this marker complements miRs-191 and miR-486-3p to make a highly predictive three-miR marker. MiRs-486-3p and miR-16 make a complementary CCA diagnosis more than any other marker pair, and therefore these miRs combine to have the highest predictive value of any two-miR marker (sensitivity $57 \%$ and specificity $98 \%$ ). Only $C C A 3$ is accurately classified by four miRs, and no CCA samples are accurately classified by all five miRs from Figure 5.

The origin of high specificity among the most predictive markers from Table $3 \mathrm{C}$ is shown in Figure 5C and 5D. For example, miR-486-3p never makes a false-positive classification. MiRs - 16 and miR-1274b both classify CTRL37 as CCA; this is the only false-positive classification for either miR-16 or 1274b. Similarly, miR-484 makes only a single falsepositive classification (CTRL5). And, miR-191 makes two false-positive classifications, which are coincident with miRs-16 and miR-1274b in classifying CTRL37 as CCA and with miR-484 in classifying CTRL5 as CCA. Because these five miRs rarely make a falsepositive classification, and because they are complementary in making true-positive classifications, MOCA was able to combine them into multi-miR biomarkers with reasonably well-balanced predictive value.

\section{Discussion}

The field of CCA is in urgent need of better diagnostic methods. While the overall survival of CCA patients is dismal, there is a large discrepancy between survival of patients diagnosed early and the vast majority of patients, who are diagnosed late in their disease. The data presented herein delineates a 5-miR panel with superior diagnostic accuracy for CCA when compared to the currently available diagnostic methods. In addition, these studies are the first to identify and characterize EVs in human bile. The presence of miR- 
laden EVs in human bile has physiologic as well as potential pathologic implications. It was recently demonstrated that hepatocellular carcinoma (HCC) cells release EVs rich in miR species, which are believed to function in intrahepatic cell-cell signaling (20). In addition, it was shown that exosomes, a type of extracellular vesicles, exist in rat bile, interact with cholangiocytes, and are able to modulate intracellular growth mechanisms (21). These findings suggest a new paradigm, wherein liver and biliary tree cells communicate through EVs and extracellular vesicle-transported miR species $(21,22)$. Our study adds to this paradigm and is the first to put forward the hypothesis that human bile acts as a physiologic and pathologic conduit allowing the communication of information, in the form of extracellular vesicle-transported miR species, between various cells within the liver and biliary tree. These findings open a broad new avenue of investigation for understanding normal physiologic signaling, as well as potential implications in disease, such as CCA.

The current study furnishes strong evidence that RNA isolated from human bile derives from free-floating cells, as well as biliary EVs. Although our vesicle isolation protocol is geared towards exosome isolation through employment of differential ultracentrifugation, further studies are needed to definitely conclude that our findings are specific for exosomes, and not extracellular vesicles in general. In addition, this study establishes that RNA originating from these free-floating cells is rapidly degraded, both by storing bile at room temperature (even for 1 hour), and by a single freeze-thaw cycle. We conclude that any bilebased miR panel developed from whole, cell-containing bile will be unpredictably biased by bile processing, and therefore destined to have limited clinical applicability (7). In contrast, we demonstrate that bile EVs contain abundant miR species that are stable and therefore usable for the development of bile-derived miR-based diagnostic panels.

Utilization of bodily fluids for the development of disease markers is appealing, since these fluids can be obtained via non-invasive (urine), minimally invasive (blood), or moderately invasive (bile) procedures. Nevertheless, the mere presence of EVs in these bodily fluids, along with the fact that they contain miR species, is not sufficient to develop stable, reproducible diagnostic panels. The field of miR-based disease markers is plagued by contradictory and often irreproducible results (9). An intuitive explanation for this problem is a lack of standardization in specimen collection, storage and processing. An additional difficulty is normalization of miR values in biologic fluids. In contrast to tissues, biologic fluids have little long RNA species (such as mRNA), and these species are quickly degraded. Therefore, attempts to normalize to a standard housekeeping gene are destined to failure. A multitude of biologic fluid-derived miR panels, including the sole bile-based miR panel published to date (7), utilize as normalizer the small RNA, U6. However, there is a growing body of evidence suggesting that utilization of U6 to normalize miR expression in body fluids introduces biases that have the potential of rendering the results un-reproducible and/or unusable in a clinical laboratory setting (9). Although there is no indication that any other intrinsic RNA species performs better than U6, there is accumulating evidence that U6 is not appropriate as a normalizer $(10,11)$. In addition, the use of any other intrinsic miR species as a normalizer is fundamentally based on the assumption that such a miR with constant expression across physiologic and pathologic states exists. There is no evidence to date to suggest that such a miR species exists in biliary EVs. Presently, there is no data to suggest that any intrinsic RNA normalizer, whose expression is stable in normal and more 
importantly in diseased states, exists in biologic fluids. Therefore, we propose that biologic fluid-derived miR levels should be normalized to initial volume of fluid analyzed. In addition, our study establishes that RNA extraction efficiency varies significantly among specimens, arguing that studies lacking a spike-in control may produce unreliable results.

Cancer diagnosis and clinical outcome prediction studies have created a relatively new field for applied mathematics. However, many previous studies have been limited to a single analytic method. Our current study compares SVMs and RF, two of the most commonly used mathematical models for cancer diagnosis (23), to MOCA, a recently developed mathematical model. One limitation of SVMs and RF is a lack of transparency in the classifiers that result from training. Because these models are "black boxes", it is difficult to apply real biological principles to subsequent clinical testing, and continued use of the initial training model is required. Conversely, MOCA returned the combination of miR species that optimized CCA diagnosis, and the predictive value that each miR contributed to that combined biomarker. Furthermore, MOCA returned the same biomarkers regardless of how the 10-fold cross-validation data was split; SVM and RFs varied as a function of data split, and for RFs the model was dependent on the "seed" employed (see Materials and Methods).

We deliberately tuned our analytic technique for high specificity, since the clinical consequences of false-positive CCA diagnosis would be calamitous. With specificity at $96 \%$, our 5-miR panel displayed a sensitivity of $67 \%$. Thus, the overall performance of this panel is superior to CA 19-9, as well as to any CCA diagnostic method currently employed in clinical practice. Further studies are needed to verify these findings in larger cohorts and to explore the potential utility of combining our new 5-miR panel with other marker panels or with established clinical modalities, such as cytology.

We foresee potential clinical utility of our marker panel in patients with obstruction in the biliary tree (Supplementary Table 1). The typical patient who would benefit from this marker panel is a PSC patient. In our analyses, 12 of 13 patients with PSC and no cancer were correctly diagnosed as not having CCA. Please note that all patients were followed for 5 years since the date the bile was collected, to ensure that at least for 5 years they would not develop CCA. The PSC patient who was diagnosed with CCA continues to be followed at our hospital. There is still no evidence of CCA. The 2 potential explanations for this seemingly false positive result are: 1 . The method is not perfect and the results need to be integrated clinically and with other laboratory results and 2 . The biologic state that induced a positive test is reversed, or reversible. Further studies are needed in order to answer this question.

Among patients with a known diagnosis of CCA, four had a preexisting diagnosis of PSC. Three of these 4 were diagnosed correctly by our marker panel ( Supplementary Table 3). The single patient misdiagnosed as CCA-negative did not have CA19-9 levels drawn. Of the remaining 3 patients correctly diagnosed by our marker panel, two had CA19-9 levels recorded. One of these had a CA19-9 level of 574.6 units $/ \mathrm{mL}$, suggestive of CCA. However, the other had a CA19-9 level of only 77.8 units $/ \mathrm{mL}$, which although above the upper normal limit, is below the accepted cutoff value of 129 units $/ \mathrm{mL}$ currently utilized to diagnose CCA (24).

Hepatology. Author manuscript; available in PMC 2015 September 01. 
Our marker panel outperformed CA19-9 levels in non-PSC patients. Among 39 CCA patients with recorded CA19-9 values (Table 1B), our marker panel correctly diagnosed 28, translating to a sensitivity of $71 \%$. The accepted cutoff CA19-9 value of 100 units/ML (in patients without PSC) correctly diagnosed only 23 patients, for a sensitivity of only $58 \%$ (2). This calculated sensitivity of CA19-9 is slightly higher than, but similar to, that found in a previous study (53\%).Thus, among every 100 CCA patients, our method is expected to diagnose 13 patients more than would CA19-9 levels. Notably, among 11 CCA patients correctly diagnosed by our 5-miR panel but misdiagnosed by CA19-9, there were 8 CCA patients without lymph node or distant metastatic implants (NOM0). These patients are potentially cured by resection. In contrast, of the 6 patients correctly diagnosed with CCA by CA19-9 but not by our 5-miR panel, only 2 were NOM0 ( Supplementary Table 4). In further support of our hypothesis that our panel can better identify early tumors, the two T1N0M0 cancers in our cohort were correctly diagnosed by our panel, but not by CA19-9 levels. Diagnosing these early cancers is crucial, since the only current curative option for CCA patients is surgery, which can only be performed early in the course of this disease. We conclude that CA 19-9 tends to diagnose advanced CCA, for which surgery may no longer be an option, while our marker panel tends to diagnose early CCA, where surgery is still an option. Therefore, the difference in sensitivity between our marker panel and CA19-9 levels ( $71 \%$ vs. $58 \%$ ) may actually underestimate our marker panel's potential impact on patient survival. Clearly, prospective studies are needed to answer this important question. Finally, in our 39-patient CCA cohort, 36 were correctly diagnosed by either our marker panel or CA 19-9, translating into a combined sensitivity of $89.7 \%$. Thus, even if treatments for advanced CCA improve, combining our marker panel with CA19-9 levels may become a valuable diagnostic strategy.

\section{Supplementary Material}

Refer to Web version on PubMed Central for supplementary material.

\section{Acknowledgments}

Financial support:

This study was supported by a K08 Award (DK090154-01) from the NIH (to F.M.S.) and by an Early Research and Detection Network (EDRN) Associate Membership supported by an U01 Award (CA086402) from the NIH. Dr. Meltzer is American Cancer Society Clinical Research Professor.

\section{Abbreviations}

$\begin{array}{ll}{ }^{\circ} \mathbf{C} & \text { Celsius } \\ \text { CA19-9 } & \text { carbohydrate antigen 19-9 } \\ \text { CCA } & \text { Cholangiocarcinoma } \\ \text { CP } & \text { chronic pancreatitis } \\ \text { CT } & \text { Computed tomography } \\ \text { Ct } & \text { Cycle passing threshold }\end{array}$




\begin{tabular}{ll} 
CTRL & Control \\
dCCA & Distal cholangiocarcinoma \\
ERCP & Endoscopic retrograde cholangiopancreatography \\
EV & extracellular vesicle \\
EUS & Endoscopic ultrasound \\
FDR & False-discovery rate \\
HCC & Hepatocellular carcinoma \\
iCCA & Intrahepatic cholangiocarcinoma \\
IR & Interventional radiology \\
IRB & Institutional Review Board \\
MRI & Magnetic resonance imaging \\
HG & Micrograms \\
miR & microRNA \\
MOCA & Multivariate Organization of Combinatorial Alterations \\
nm & Nanometer \\
NTA & Nanoparticle tracking analysis \\
pCCA & Perihilar cholangiocarcinoma \\
PTA & Phosphotungstic acid \\
RF & Random Forests \\
RIPA & Radio-immunoprecipitation assay \\
SOD & Sphincter of Oddi dysfunction \\
SSP411 & Spermatogenesis associated 20 \\
SVMs & Support Vector Machines \\
TEM & Transmission electron microscopy \\
TSG101 & Tumor Susceptibility Gene 101 \\
U & Union \\
\hline
\end{tabular}

\section{References}

1. Razumilava N, Gores GJ. Classification, diagnosis, and management of cholangiocarcinoma. Clin Gastroenterol Hepatol. 2013; 11:13-21. e11; quiz e13-14. [PubMed: 22982100]

2. Blechacz B, Komuta M, Roskams T, Gores GJ. Clinical diagnosis and staging of cholangiocarcinoma. Nat Rev Gastroenterol Hepatol. 2011; 8:512-522. [PubMed: 21808282]

3. Patel T. Cholangiocarcinoma--controversies and challenges. Nat Rev Gastroenterol Hepatol. 2011; 8:189-200. [PubMed: 21460876]

Hepatology. Author manuscript; available in PMC 2015 September 01. 
4. Rimola J, Forner A, Reig M, Vilana R, de Lope CR, Ayuso C, Bruix J. Cholangiocarcinoma in cirrhosis: absence of contrast washout in delayed phases by magnetic resonance imaging avoids misdiagnosis of hepatocellular carcinoma. Hepatology. 2009; 50:791-798. [PubMed: 19610049]

5. Scarlett CJ, Saxby AJ, Nielsen A, Bell C, Samra JS, Hugh T, Baxter RC, et al. Proteomic profiling of cholangiocarcinoma: diagnostic potential of SELDI-TOF MS in malignant bile duct stricture. Hepatology. 2006; 44:658-666. [PubMed: 16941699]

6. Chapman MH, Tidswell R, Dooley JS, Sandanayake NS, Cerec V, Deheragoda M, Lee AJ, et al. Whole genome RNA expression profiling of endoscopic biliary brushings provides data suitable for biomarker discovery in cholangiocarcinoma. J Hepatol. 2011; 56:877-885. [PubMed: 22173169]

7. Shigehara K, Yokomuro S, Ishibashi O, Mizuguchi Y, Arima Y, Kawahigashi Y, Kanda T, et al. Real-time PCR-based analysis of the human bile microRNAome identifies miR-9 as a potential diagnostic biomarker for biliary tract cancer. PLoS ONE. 2011; 6:e23584. [PubMed: 21858175]

8. Kern SE. Why your new cancer biomarker may never work: recurrent patterns and remarkable diversity in biomarker failures. Cancer Res. 2012; 72:6097-6101. [PubMed: 23172309]

9. Cortez MA, Bueso-Ramos C, Ferdin J, Lopez-Berestein G, Sood AK, Calin GA. MicroRNAs in body fluids--the mix of hormones and biomarkers. Nat Rev Clin Oncol. 2011; 8:467-477. [PubMed: 21647195]

10. Chen X, Ba Y, Ma L, Cai X, Yin Y, Wang K, Guo J, et al. Characterization of microRNAs in serum: a novel class of biomarkers for diagnosis of cancer and other diseases. Cell Res. 2008; 18:997-1006. [PubMed: 18766170]

11. Hummel R, Hussey DJ, Haier J. MicroRNAs: predictors and modifiers of chemo- and radiotherapy in different tumour types. Eur J Cancer. 2010; 46:298-311. [PubMed: 19948396]

12. Mitchell PS, Parkin RK, Kroh EM, Fritz BR, Wyman SK, Pogosova-Agadjanyan EL, Peterson A, et al. Circulating microRNAs as stable blood-based markers for cancer detection. Proc Natl Acad Sci U S A. 2008; 105:10513-10518. [PubMed: 18663219]

13. Lasser C, Eldh M, Lotvall J. Isolation and characterization of RNA-containing exosomes. J Vis Exp. 2012:e3037. [PubMed: 22257828]

14. Masica DL, Karchin R. Collections of simultaneously altered genes as biomarkers of cancer cell drug response. Cancer Res. 2013; 73:1699-1708. [PubMed: 23338612]

15. Masica DL, Karchin R. Correlation of somatic mutation and expression identifies genes important in human glioblastoma progression and survival. Cancer Res. 2011; 71:4550-4561. [PubMed: 21555372]

16. Silverman JM, Reiner NE. Exosomes and other microvesicles in infection biology: organelles with unanticipated phenotypes. Cell Microbiol. 2011; 13:1-9. [PubMed: 21040357]

17. Mathivanan S, Ji H, Simpson RJ. Exosomes: extracellular organelles important in intercellular communication. J Proteomics. 2010; 73:1907-1920. [PubMed: 20601276]

18. Ohno S, Takanashi M, Sudo K, Ueda S, Ishikawa A, Matsuyama N, Fujita K, et al. Systemically injected exosomes targeted to EGFR deliver antitumor microRNA to breast cancer cells. Mol Ther. 2013; 21:185-191. [PubMed: 23032975]

19. Gallo A, Tandon M, Alevizos I, Illei GG. The majority of microRNAs detectable in serum and saliva is concentrated in exosomes. PLoS ONE. 2012; 7:e30679. [PubMed: 22427800]

20. Kogure T, Lin WL, Yan IK, Braconi C, Patel T. Intercellular nanovesicle-mediated microRNA transfer: a mechanism of environmental modulation of hepatocellular cancer cell growth. Hepatology. 2011; 54:1237-1248. [PubMed: 21721029]

21. Masyuk AI, Huang BQ, Ward CJ, Gradilone SA, Banales JM, Masyuk TV, Radtke B, et al. Biliary exosomes influence cholangiocyte regulatory mechanisms and proliferation through interaction with primary cilia. Am J Physiol Gastrointest Liver Physiol. 2010; 299:G990-G999. [PubMed: 20634433]

22. Witek RP, Yang L, Liu R, Jung Y, Omenetti A, Syn WK, Choi SS, et al. Liver cell-derived microparticles activate hedgehog signaling and alter gene expression in hepatic endothelial cells. Gastroenterology. 2009; 136:320-330. e322. [PubMed: 19013163]

23. Burdick E, Falotico-Taylor J, Young JM. Technology assessment in the Coronary Artery Surgery Study. Int J Technol Assess Health Care. 1991; 7:171-181. [PubMed: 1864701] 
24. Levy C, Lymp J, Angulo P, Gores GJ, Larusso N, Lindor KD. The value of serum CA 19-9 in predicting cholangiocarcinomas in patients with primary sclerosing cholangitis. Dig Dis Sci. 2005; 50:1734-1740. [PubMed: 16133981]

25. Patel AH, Harnois DM, Klee GG, LaRusso NF, Gores GJ. The utility of CA 19-9 in the diagnoses of cholangiocarcinoma in patients without primary sclerosing cholangitis. Am J Gastroenterol. 2000; 95:204-207. [PubMed: 10638584] 
A.

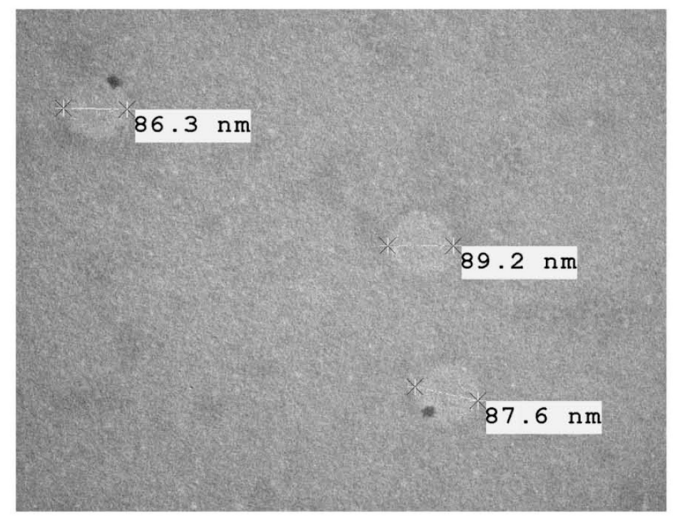

B.

Tsg101 (43 KDa)

CD63 (53 KDa)
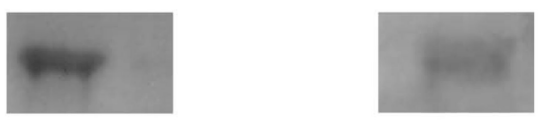

C.

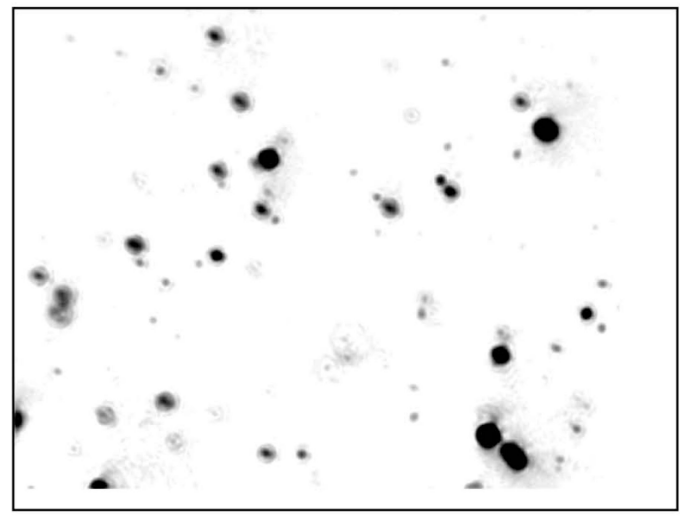

D.

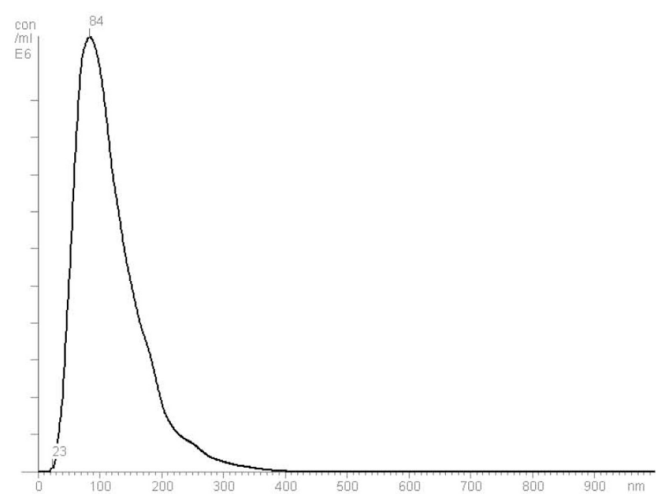

Figure 1. Human biliary EVs characterization

Panel $\boldsymbol{A}$ includes a typical TEM picture demonstrating the presence of $30-110 \mathrm{nM}$ spherical structures in human bile. Further characterization demonstrates that these vesicles display extracellular vesicle characteristics. Panel $\boldsymbol{B}$ demonstrates presence of typical extracellular vesicle proteins (TSG101 and CD63) in EV preparations from human bile. Panel $C$ demonstrates the same 30-110 nM vesicles as visualized with NTA. Panel $\boldsymbol{D}$ demonstrates that the mode of EVs isolated from human bile was determined to be approximately $84 \mathrm{~nm}$ (X-axis depicts EVs size and Y-axis depicts EVs concentration for each size). 

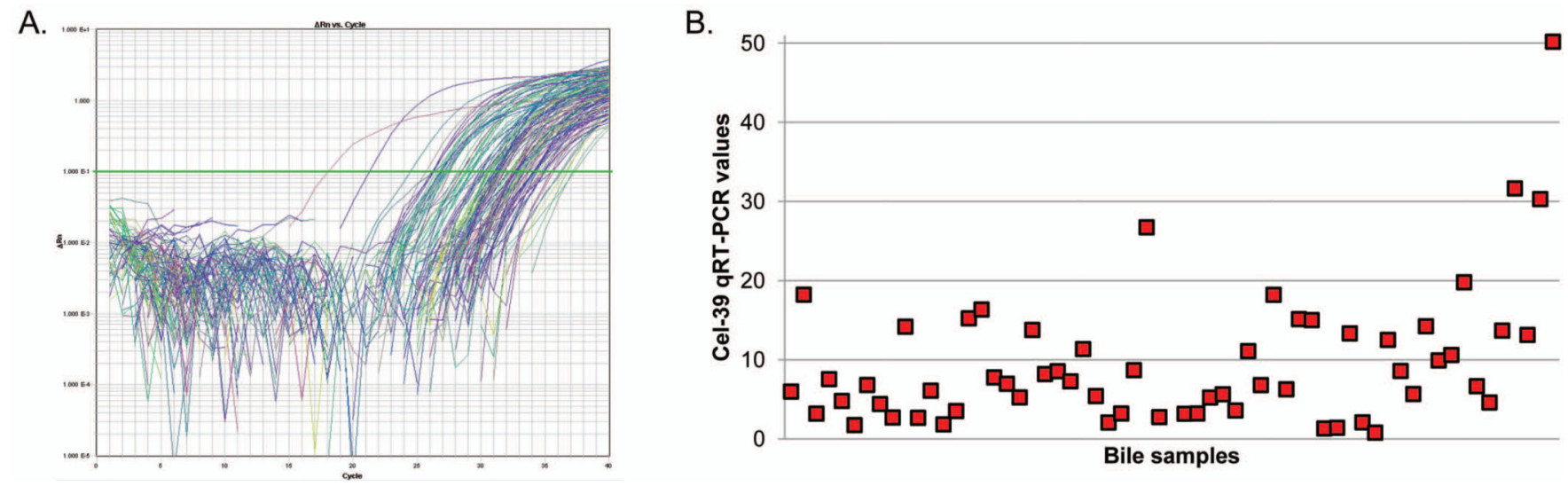

Figure 2. miR species extraction from human bile extracellular vesicles Panel $\boldsymbol{A}$ shows amplification curves for miR species from EVs isolated from a bile specimen (X-axis - Cycle number and Y-axis - measured miR expression). Panel $\boldsymbol{B}$ depicts the large variability in measured Cel-miR-39 quantity after Cel-miR-39 was spiked at equal concentration in a cohort of 60 bile specimens (X-axis - bile specimens, $\mathrm{Y}$-axis - measured qRT-PCR value for Cel-miR-39). 

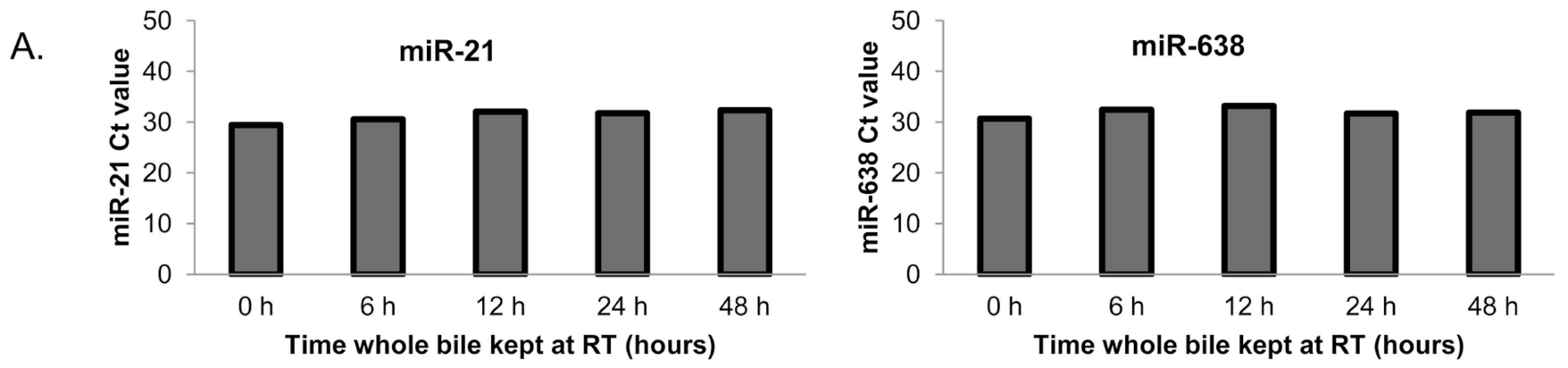

B.
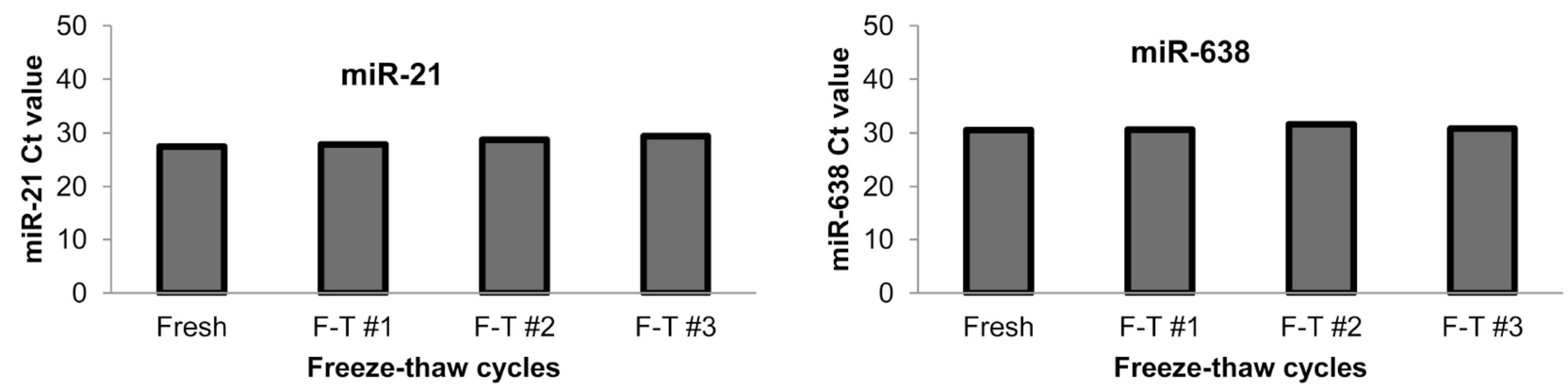

Figure 3. High stability and reproducibility of measured miR expression in bile EVs extracts Panel $\boldsymbol{A}$ demonstrates that the levels of miR-21 and miR-638 are stable in EVs isolated from human bile kept at RT for 2-24 hours. Panel $\boldsymbol{B}$ demonstrates the stability of miR-21 and miR-638 after multiple freeze-thaw cycles. 


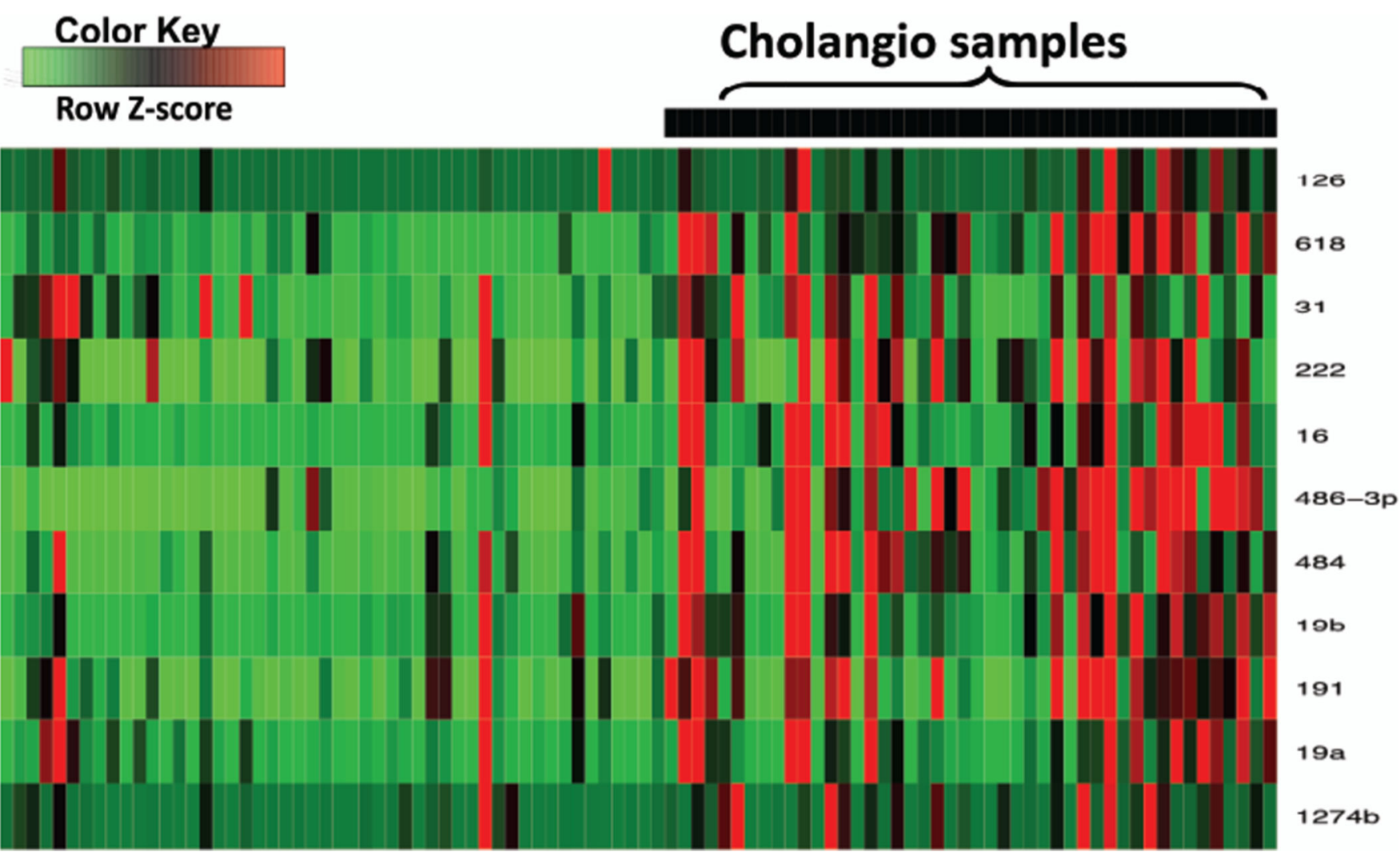

Figure 4. Expression of 11 selected miR species across 96 samples

The heatmap segregates the 50 control samples (left side) and 46 CCA samples (right side). Bright red was used for all coordinates with a Z-score $\geq 0.5$; therefore, these coordinates correspond to a CCA classification by MOCA. See Table 3B for mean expression values and diagnostic thresholds for all $11 \mathrm{miR}$ species. 
A

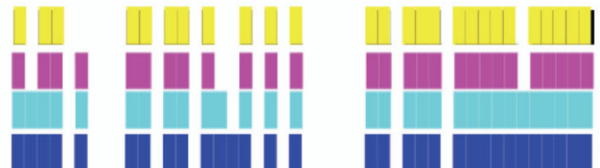

B

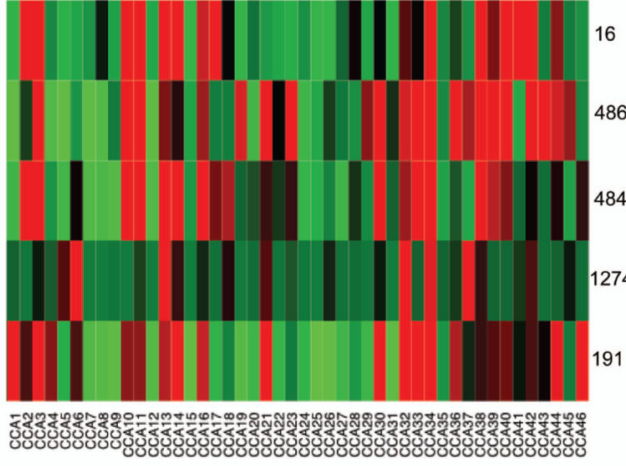

191 4 486-3p

$191 \cup 486-3 \mathrm{p} U 1274 \mathrm{~b}$

$191 \cup 486-3 \mathrm{p} \cup 1274 \mathrm{~b} \cup 16$

86-3p

484

$274 b$

191

Color Key

Row Z-score
C
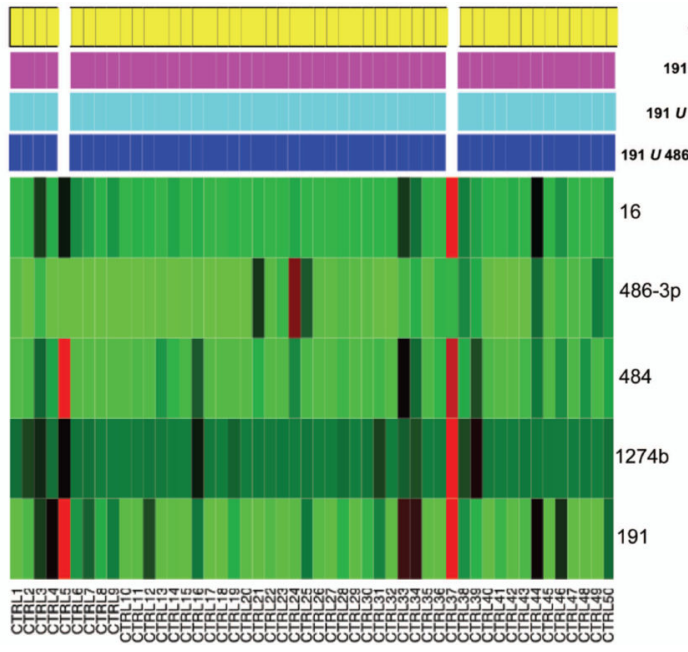

191 U 486-3p

191 U 486-3p U 1274b

$191 \cup 486-3 \mathrm{p} U 1274 \mathrm{~b} U 16$

$\cup 486-3 p \cup 1274 b \cup 16 \cup 48$

Figure 5. Bile specimen classification by multi-miR markers with high predictive value Panels $\boldsymbol{A}$ and $\boldsymbol{B}$ display classification across 46 CCA samples by each of the markers from Table 3C (color bars in $A$ ) and expression for each of the corresponding miRs (heatmap in $B$ ). In $B$, bright red was used for all coordinates with a Z-score $\geq 0.5$; therefore, these coordinates correspond to a CCA classification by MOCA. For each multi-miR marker, panel A displays the correct diagnosis with a solid rectangle. Panels $\boldsymbol{C}$ and $\boldsymbol{D}$ display classification across 50 control (CTRL) samples by each of the markers from Table 3C. For each multi-miR marker, panel D displays the correct diagnosis with a solid rectangle 


\begin{tabular}{|c|c|c|c|c|c|c|c|c|c|c|c|c|c|c|c|c|c|c|c|c|c|c|c|c|c|c|c|}
\hline & $\sum_{i}$ & 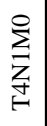 & $\mid \begin{array}{l}0 \\
\sum_{0} \\
\sum_{\tilde{m}} \\
\hat{f}\end{array}$ & 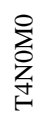 & 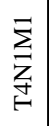 & $\sum_{\substack{n \\
\sum_{n}}}^{0}$ & 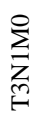 & $\sum_{Z}^{\bar{Z}}$ & 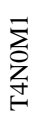 & 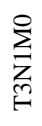 & 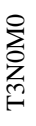 & 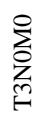 & 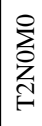 & 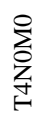 & 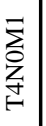 & 离 & 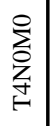 & 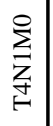 & 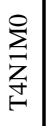 & 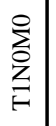 & $\sum_{\substack{n \\
\oplus}}^{\infty}$ & 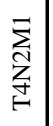 & 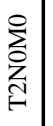 & 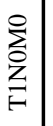 & 亲 & 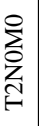 & $\begin{array}{l}\sum_{0}^{\circ} \\
\stackrel{2}{m}\end{array}$ \\
\hline & 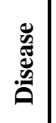 & $\begin{array}{l}\mathbb{U} \\
\text { Z }\end{array}$ & త্ঠي & $\begin{array}{l}\mathbb{U} \\
\text {. }\end{array}$ & 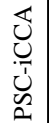 & 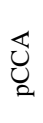 & $\begin{array}{l}\mathbb{U} \\
\mathbb{Z}\end{array}$ & ِيْ & $\begin{array}{l}\mathbb{U} \\
.0\end{array}$ & త্ర్ & 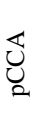 & 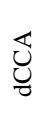 & $\begin{array}{l}\mathbb{U} \\
\text {. }\end{array}$ & $\begin{array}{l}\mathbb{U} \\
.\end{array}$ & 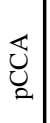 & త্రي & $\begin{array}{l}\mathbb{U} \\
\text {. }\end{array}$ & $\begin{array}{l}\mathbb{U} \\
0 \\
0\end{array}$ & $\begin{array}{l}\mathbb{U} \\
\text { }\end{array}$ & త্రي & $\begin{array}{l}\mathbb{U} \\
己 \\
2\end{array}$ & త্ర్ & $\begin{array}{l}\mathbb{U} \\
\underset{Z}{2}\end{array}$ & 范 & త్ర & 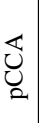 & $\begin{array}{l}\mathbb{U} \\
\text {. }\end{array}$ \\
\hline & 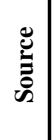 & 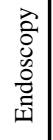 & $\cong$ & $\cong$ & 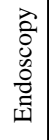 & 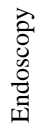 & 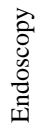 & 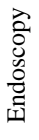 & $\cong$ & 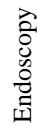 & $\cong$ & $\cong$ & $\cong$ & $\cong$ & $\cong$ & $\cong$ & $\cong$ & 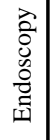 & $\cong$ & $\cong$ & 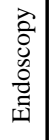 & 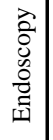 & 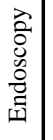 & $\cong$ & 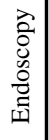 & $\cong$ & $\cong$ \\
\hline & $\frac{\hat{d}}{0}$ & $\begin{array}{l}\stackrel{\partial}{\dot{y}} \\
\dot{y}\end{array}$ & $\stackrel{\overbrace{}}{\gtrless}$ & $\bar{\infty}$ & $\overleftrightarrow{z}$ & ڤ̊ & 恣 & $\begin{array}{l}0 \\
\dot{d}\end{array}$ & $\overleftrightarrow{z}$ & बे. & 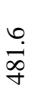 & $\vec{\infty}$ & 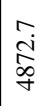 & $\hat{\stackrel{\circ}{\circ}}$ & 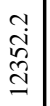 & $\underset{\text { in }}{\stackrel{\infty}{\vec{n}}}$ & $\begin{array}{l}\text { î. } \\
\text { ठ̀. }\end{array}$ & 孞 & 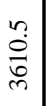 & $\bar{\infty}$ & - & 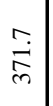 & $\stackrel{6}{\sigma}$ & $\vec{\infty}$ & \begin{tabular}{l}
\multirow{2}{0}{} \\
ఫे
\end{tabular} & o: & ठे \\
\hline & 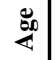 & in & in & in & $\tilde{m}$ & 8 & $\infty$ & 'f & $\mathscr{\infty}$ & $\approx$ & 8 & 8 & t & in & $\stackrel{n}{2}$ & $\stackrel{\infty}{\infty}$ & $\bar{n}$ & 8 & $\approx$ & 6 & 8 & 8 & in & $\delta$ & 于 & 8 & $\overline{6}$ \\
\hline & 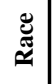 & 0 & U & U & $\mathbb{4}$ & $\varangle$ & $u$ & U & $\mathbb{4}$ & u & U & $u$ & $\Psi$ & $u$ & u & 0 & $u$ & $u$ & $u$ & u & u & $u$ & $u$ & u & u & $\mathbb{4}$ & $u$ \\
\hline & 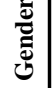 & $\Sigma$ & $\Sigma$ & I & I & $I$ & $\Sigma$ & $\Sigma$ & $\amalg$ & $\Sigma$ & 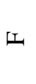 & $\Sigma$ & $\Sigma$ & $\Phi$ & I & II & $\Sigma$ & $\Sigma$ & 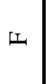 & 工 & $\amalg$ & 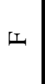 & $\amalg$ & 工 & $\Phi$ & $\Sigma$ & $\omega_{1}$ \\
\hline 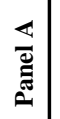 & 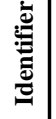 & 苂 & 效 & 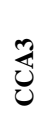 & 胥 & 先 & 离 & త্ & 罜 & 己े & 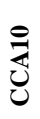 & ত্য & $\frac{\tilde{J}}{\mathrm{U}}$ & $\overbrace{d}^{m}$ & 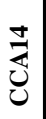 & $\begin{array}{l}\frac{10}{3} \\
\frac{u}{U}\end{array}$ & 苞 & 矛 & 热 & $\begin{array}{l}\overrightarrow{\vec{t}} \\
\vec{U}\end{array}$ & త్ & 离 & $\begin{array}{l}\tilde{y} \\
\mathbb{U} \\
0\end{array}$ & త্য & $\begin{array}{l}\text { J } \\
\text { U̦ }\end{array}$ & 瓷 & 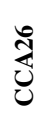 \\
\hline
\end{tabular}




\begin{tabular}{|c|c|c|c|c|c|c|c|c|c|c|c|c|c|c|c|c|c|c|c|c|c|c|c|c|c|c|c|c|}
\hline & $\sum_{i}$ & 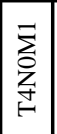 & 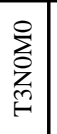 & 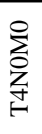 & 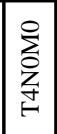 & 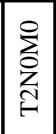 & 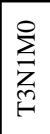 & 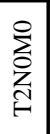 & 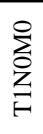 & 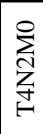 & 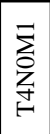 & 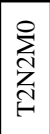 & 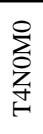 & 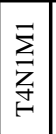 & 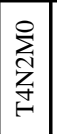 & 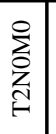 & $\sum_{\substack{\mid \\
\oplus}}^{=}$ & 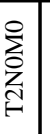 & 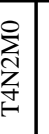 & 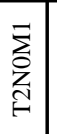 & 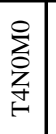 & & & & & & & \\
\hline & 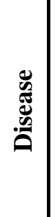 & $\begin{array}{l}\mathbb{U} \\
0 ِ=\end{array}$ & 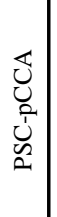 & $\begin{array}{l}\mathbb{Z} \\
\text {. }\end{array}$ & $\begin{array}{l}\mathbb{J} \\
\text {. }\end{array}$ & $\begin{array}{l}\mathbb{U} \\
\text { Z }\end{array}$ & 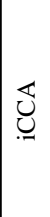 & $\begin{array}{l}\mathbb{U} \\
\text {. }\end{array}$ & త্రু & ర্ & 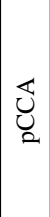 & త্ర & 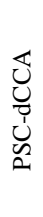 & 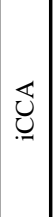 & 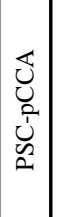 & $\begin{array}{l}\mathbb{J} \\
0 \\
0\end{array}$ & 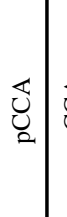 & 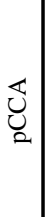 & $\begin{array}{l}U_{0} \\
\text {. }\end{array}$ & $\begin{array}{l}\mathbb{U} \\
\mathbb{Z}\end{array}$ & & 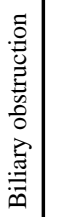 & 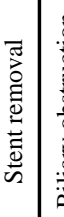 & & 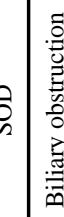 & | & 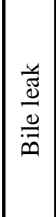 & 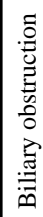 \\
\hline & 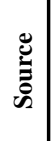 & $\cong$ & $\begin{array}{l}\overrightarrow{0} \\
\overline{0} \\
0 \\
0 \\
0 \\
0\end{array}$ & 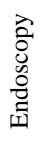 & $\cong$ & 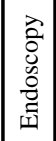 & 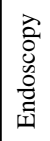 & $\cong$ & $\cong$ & $\cong$ & $\begin{array}{l}\hat{0} \\
\overline{0} \\
0 \\
0 \\
\text { : }\end{array}$ & 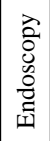 & 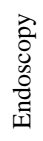 & 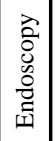 & 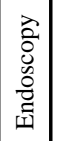 & 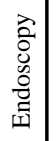 & $\begin{array}{l}\text { aे } \\
0 \\
0 \\
0 \\
0 \\
0\end{array}$ & 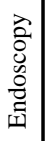 & $\begin{array}{l}\hat{0} \\
0 \\
0 \\
0 \\
0 \\
0\end{array}$ & $\cong$ & 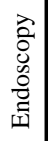 & $\begin{array}{l}\text { aे } \\
0 \\
\text { : } \\
\text { : }\end{array}$ & 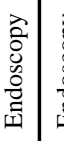 & 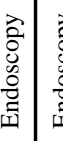 & 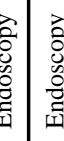 & 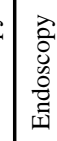 & 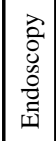 & 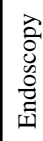 \\
\hline & فे & $\mathbb{z}$ & $\overleftrightarrow{z}$ & $\stackrel{\curvearrowright}{\curvearrowright}$ & $\stackrel{m}{\stackrel{2}{*}}$ & 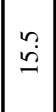 & $\begin{array}{l}0 \\
\substack{\infty \\
i}\end{array}$ & $\stackrel{\odot}{\vec{v}}$ & $\overleftrightarrow{z}$ & $\overleftrightarrow{z}$ & $\begin{array}{l}\dot{d} \\
\text { d్ }\end{array}$ & $\begin{array}{l}\vec{\partial} \\
\vec{\lambda}\end{array}$ & $\stackrel{\infty}{\stackrel{\infty}{\Sigma}}$ & $\begin{array}{l}\dot{r} \\
\stackrel{\dot{m}}{g}\end{array}$ & $\begin{array}{l}0 \\
\dot{+} \\
\text { in }\end{array}$ & $\stackrel{n}{n}$ & $\nabla$ & $\mathbb{z}$ & $\stackrel{+}{\stackrel{+}{\Xi}}$ & $\stackrel{\partial}{\hat{\sigma}}$ & $\begin{array}{l}m \\
\stackrel{2}{0} \\
0\end{array}$ & $\overleftrightarrow{z}$ & $\overleftrightarrow{z}$ & $\overleftrightarrow{z}$ & 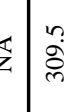 & 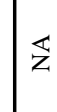 & $\overleftrightarrow{\mathrm{z}}$ & 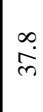 \\
\hline & $\frac{80}{4}$ & 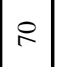 & ले & $\stackrel{\infty}{\circ}$ & in & $i$ & 6 & $\tilde{6}$ & 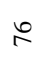 & $\beta$ & $F$ & $\infty$ & $\approx$ & $\approx$ & 8) & in & 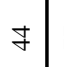 & $\approx$ & $\stackrel{\circ}{n}$ & \& & $\mathscr{q}$ & $\stackrel{2}{ }$ & in & $\infty$ & $n$ & $\bar{\sigma}$ & in & $\stackrel{8}{8}$ \\
\hline & 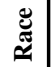 & U & ن & $\varangle$ & 0 & $x$ & U & $\mathbb{\&}$ & U & U & u & u & $\mathbb{\&}$ & $u$ & $u$ & U & $\varangle$ & u & u & u & $I$ & $I$ & U & $\mathbb{4}$ & U & U & u & 0 \\
\hline & 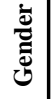 & $\Sigma$ & $\Phi$ & 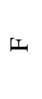 & $\Phi$ & 山 & $\Phi$ & $\Sigma$ & $\Sigma$ & $\Sigma$ & 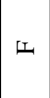 & $\Sigma$ & $\Phi$ & LI & $\Phi$ & $\Sigma$ & $\Phi$ & II & $\Sigma$ & $\Sigma$ & 山 & $\Sigma$ & $\Sigma b$ & I & 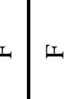 & $\mid \Sigma$ & $\Sigma$ & $\Sigma$ \\
\hline 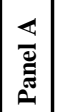 & 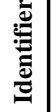 & $\mid \begin{array}{c}\hat{U} \\
\hat{U}\end{array}$ & 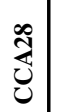 & సิ & 总 & $\overline{\vec{u}}$ & 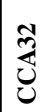 & $\stackrel{2}{2}$ & 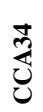 & 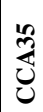 & $\frac{0}{4}$ & $\sqrt{3}$ & 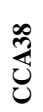 & 己ें & 导 & 忢 & \begin{tabular}{l} 
J̃ \\
\multirow{U}{*}{}
\end{tabular} & $\frac{3}{d}$ & 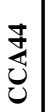 & 缹 & 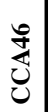 & $\overline{\vec{z}}$ & בָ & שֶ & & $\mid$ & 竎 & $\mid \frac{\infty}{\mathbb{z}}$ \\
\hline
\end{tabular}




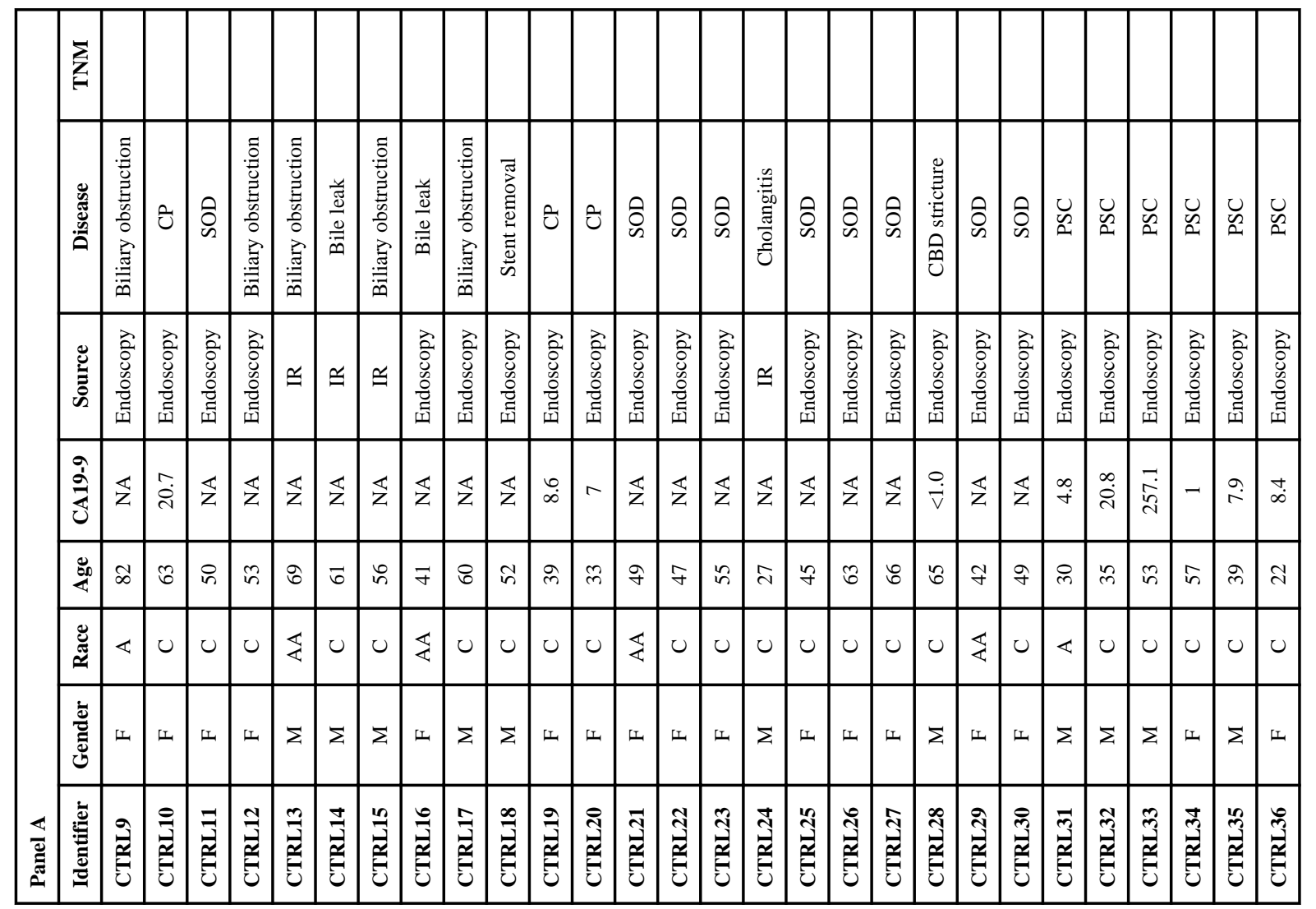




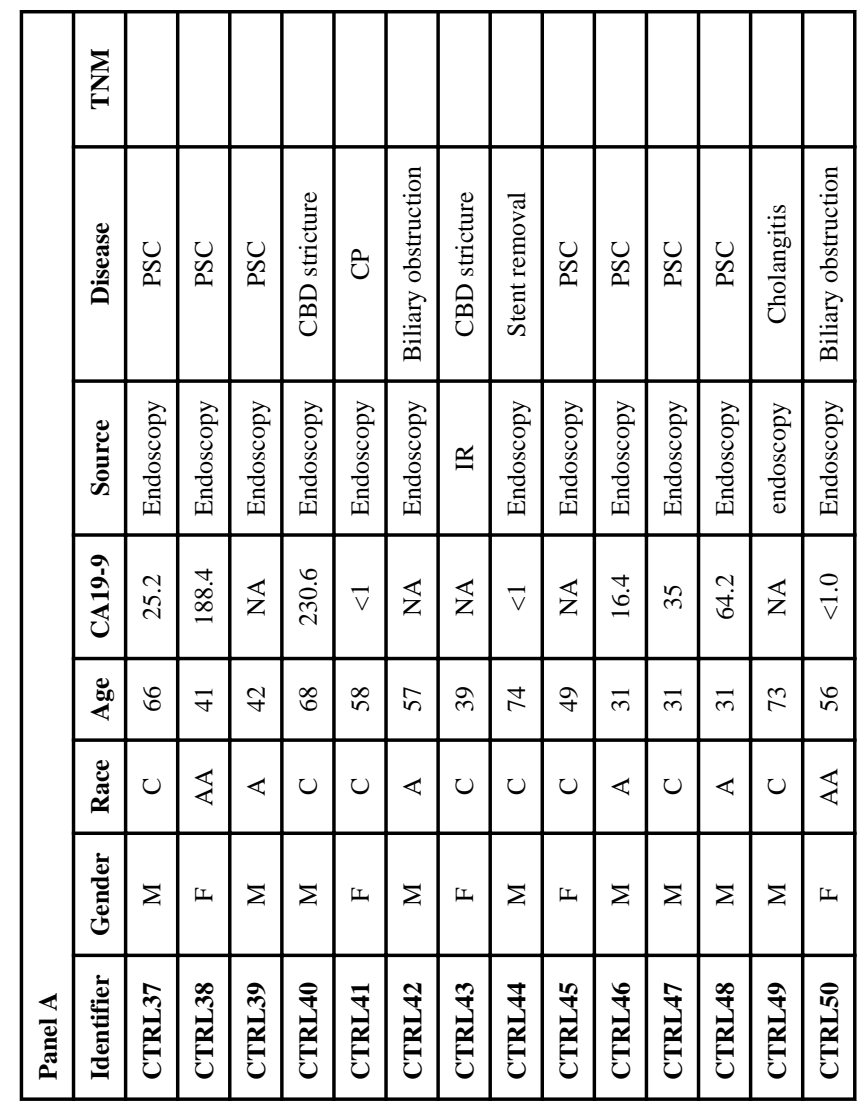

\begin{tabular}{|c|c|c|c|c|c|c|c|c|c|c|c|c|}
\hline e & - & 0 & 10 & - & - & - & - & - & 0 & - & - & - \\
\hline 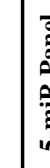 & - & - & - & 0 & - & 0 & 0 & - & - & 0 & - & - \\
\hline 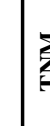 & 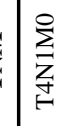 & 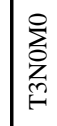 & 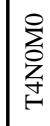 & 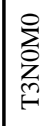 & 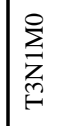 & $\underset{⿱ 亠 幺}{\sum}$ & $\sum_{\substack{\infty \\
\hat{n}}}^{\infty}$ & 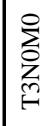 & 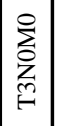 & 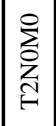 & 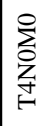 & 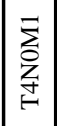 \\
\hline & 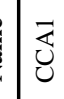 & $\tilde{U}$ & $\mathcal{U}_{0}^{2}$ & 畓 & 选 & 胥 & 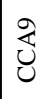 & $\mid \begin{array}{l}0 \\
\mathbb{u} \\
\text { d }\end{array}$ & $\mid \begin{array}{l}\bar{u} \\
\bar{U}\end{array}$ & $\mid \begin{array}{l}\pi \\
\mathbb{Z} \\
0\end{array}$ & 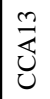 & 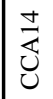 \\
\hline 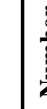 & - & $\alpha$ & $m$ & + & in & 0 & r & $\infty$ & $a$ & 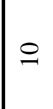 & $=$ & $\simeq$ \\
\hline & & & & & & & & & & & & \\
\hline
\end{tabular}




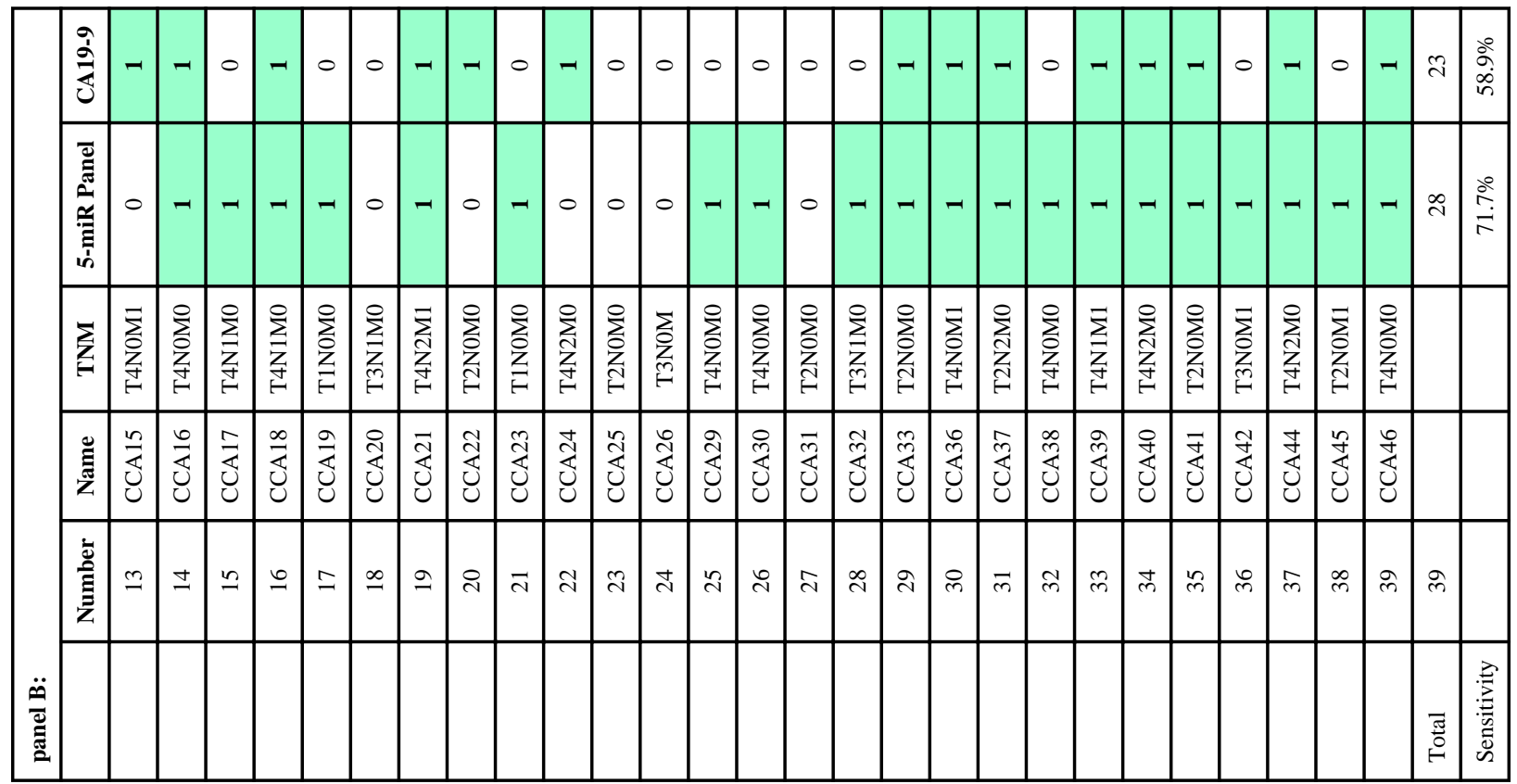




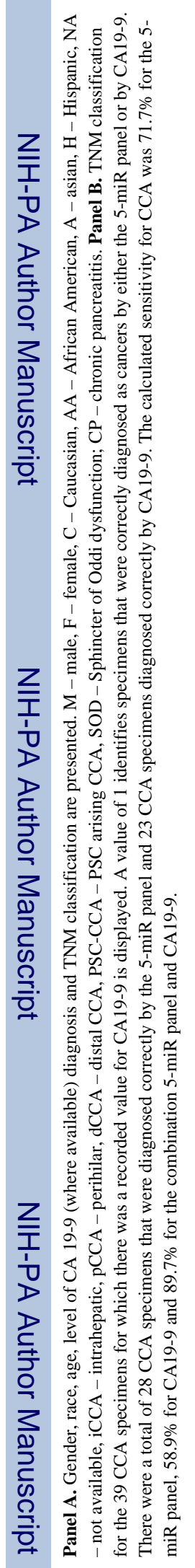

Hepatology. Author manuscript; available in PMC 2015 September 01. 


\section{Table 3}

Characteristics of mathematical approaches for data analysis.

\begin{tabular}{|c|c|c|c|c|}
\hline \multicolumn{5}{|c|}{ Panel A } \\
\hline & & Sensitivity & Specificity & \\
\hline \multicolumn{2}{|l|}{ MOCA } & $67 \%$ & $96 \%$ & \\
\hline \multicolumn{2}{|c|}{ Random Forest } & $76 \%$ & $88 \%$ & \\
\hline \multicolumn{2}{|l|}{ SVM } & $65 \%$ & $90 \%$ & \\
\hline \multicolumn{5}{|c|}{ Panel B } \\
\hline $\operatorname{miR}$ & Mean & Threshold & & \\
\hline 126 & 74.17 & 221.88 & & \\
\hline 618 & 13.3 & 26.29 & & \\
\hline 31 & 428.02 & 811.93 & & \\
\hline 222 & 35.63 & 60.88 & & \\
\hline 16 & 70.91 & 144.76 & & \\
\hline $486-3 p$ & 46.78 & 82.30 & & \\
\hline 484 & 29.26 & 54.49 & & \\
\hline $19 b$ & 66.34 & 135.98 & & \\
\hline 191 & 5.17 & 9.23 & & \\
\hline $19 \mathrm{a}$ & 41.40 & 85.70 & & \\
\hline $1274 b$ & 61.00 & 165.87 & & \\
\hline \multicolumn{5}{|c|}{ Panel C } \\
\hline & & & Sensitivity & Specificity \\
\hline \multicolumn{3}{|c|}{$191 U 486-3 \mathrm{p} U 1274 \mathrm{~b} U 16 U 484$} & $67 \%$ & $96 \%$ \\
\hline \multicolumn{3}{|c|}{$191 U 486-3 \mathrm{p} U 1274 \mathrm{~b} U 16$} & $65 \%$ & $96 \%$ \\
\hline \multicolumn{3}{|c|}{$191 U 486-3 \mathrm{p} U 1274 \mathrm{~b}$} & $59 \%$ & $96 \%$ \\
\hline \multicolumn{3}{|c|}{$191 U 486-3 \mathrm{p}$} & $57 \%$ & $96 \%$ \\
\hline
\end{tabular}

Panel $\boldsymbol{A}$ shows the predictive value achieved from each of three distinct mathematical approaches. The statistical Sensitivity and Specificity of CCA diagnosis achieved using selected miR species using the MOCA algorithm, SVM and RF. Panel $\boldsymbol{B}$ displays 11 differentially expressed miRs selected for analysis of predictive value. The table displays the $11 \mathrm{miR}$ species, the Mean expression across 96 samples for that miR, and the Threshold above which a sample would be classified as CCA by MOCA. In all cases, the threshold is significantly greater than the mean across all samples for the corresponding miR. Panel $\boldsymbol{C}$ displays markers comprising five, four, three, or two miR species and corresponding predictive value. The statistical Sensitivity and Specificity of CCA classification achieved by four, representative multi-miR markers. Multi-miR markers were combined using the union $(U)$ Boolean set operation. 